\title{
Pneumococcal polysaccharide vaccination in rheumatoid arthritis patients receiving tacrolimus
}

Kiyoshi Migita ${ }^{1,2^{*}}$, Yukihiro Akeda ${ }^{3}$, Manabu Akazawa ${ }^{4}$, Shigeto Tohma ${ }^{1}$, Fuminori Hirano ${ }^{1}$, Haruko Ideguchi ${ }^{1}$, Ryutaro Matsumura ${ }^{1}$, Eiichi Suematsu ${ }^{1}$, Tomoya Miyamura ${ }^{1}$, Shunsuke Mori ${ }^{1}$, Takahiro Fukui ${ }^{1}$, Yasumori Izumi ${ }^{1}$, Nozomi Iwanaga ${ }^{1}$, Hiroshi Tsutani ${ }^{1}$, Kouichirou Saisyo', Takao Yamanaka', Shiro Ohshima' ${ }^{1}$, Takao Sugiyama', Yojiro Kawabe', Masao Katayama', Yasuo Suenaga', Akira Okamoto', Hisaji Ohshima', Yasumasa Okada', Kenji Ichikawa', Shigeru Yoshizawa', Kenji Kawakami ${ }^{1}$, Toshihiro Matsui ${ }^{1}$, Hiroshi Furukawa ${ }^{1}$ and Kazunori Oishi ${ }^{5}$

\begin{abstract}
Introduction: In rheumatoid arthritis (RA) patients receiving immunosuppressive treatments, vaccination against Streptococcus pneumoniae is recommended. The objective of the study was to evaluate the effects of tacrolimus (TAC) on immune response following administration of a 23-valent pneumococcal polysaccharide vaccine (PPSV23) in patients with established RA.

Methods: Patients with RA $(n=133)$ were vaccinated with PPSV23. Patients were classified into TAC $(n=29)$, methotrexate (MTX) $(n=55)$, control $(n=35)$, and TAC/MTX $(n=14)$ treatment groups. We measured the concentrations of pneumococcal serotypes $6 \mathrm{~B}$ and $23 \mathrm{~F}$ by using an enzyme-linked immunosorbent assay and determined antibody functionality by using a multiplexed opsonophagocytic killing assay, reported as the opsonization index $(\mathrm{OI})$, before and 4 to 6 weeks after vaccination. A positive antibody response was defined as at least a twofold increase in the lgG concentration or as at least a 10-fold increase in the Ol.
\end{abstract}

Results: IgG concentrations and Ols were significantly increased in all treatment groups after PPSV23 vaccination. The TAC treatment group appears to respond in a manner similar to that of the RA control group in terms of $6 \mathrm{~B}$ and $23 \mathrm{~F}$ serotype concentration and function. In contrast, the MTX group had the lowest immune response. Patients who received a combination of TAC and MTX (TAC/MTX) also had a diminished immune response compared with those who received TAC alone.

Conclusions: TAC monotherapy does not appear to impair PPSV23 immunogenicity in patients with RA, whereas antibody production and function may be reduced when TAC is used with MTX. Thus, PPSV23 administration during ongoing TAC treatment should be encouraged for infection-prone TAC-treated patients with rheumatic diseases.

Trial registration: University Hospital Medical Information Network Clinical Trials Registry: UMIN000009566. Registered 12 December 2012.

\footnotetext{
*Correspondence: migita@nagasaki-mc.com

1 Japanese National Hospital Organization (NHO, EBM study group),

Higashigaoka 2-5-23, Meguro, Tokyo 152-8621, Japan

${ }^{2}$ Clinical Research Center, NHO Nagasaki Medical Center, Kubara 2-1001-1,

Omura 856-8652, Japan

Full list of author information is available at the end of the article
} 


\section{Introduction}

Patients with rheumatoid arthritis (RA) have an increased risk of developing an infectious disease as the underlying conditions and immunomodulatory therapies weaken their immune response [1]. The use of immunosuppressive agents to treat RA may also contribute to this increased risk. Therefore, routine immunizations are important to reduce infection-mediated morbidity and mortality [2, 3]. Given these concerns, the European League Against Rheumatism treatment guidelines have recommended the routine use of pneumococcal vaccines for immunecompromised patients, including those who are receiving tumor necrosis factor (TNF) inhibitors $[4,5]$. Notably, the response to vaccination varies among RA patients who are receiving TNF inhibitor therapy with or without concomitant corticosteroids or methotrexate (MTX) or both [6]. A recent meta-analysis demonstrated that patients receiving TNF inhibitors with MTX had a lower response to the pneumococcal vaccine than those receiving TNF inhibitors alone [7]. Furthermore, variable vaccine responses have been reported for patients receiving other biological agents that inhibit T-cell co-stimulation or deplete B cells with or without concomitant corticosteroid or disease-modifying antirheumatic drug (DMARD) treatment or both [8].

We previously reported that, in contrast to other biological treatment agents, tocilizumab, a humanized monoclonal antibody approved for RA treatment, does not impair the immunogenicity of the 23-valent pneumococcal polysaccharide vaccine (PPSV23) in patients with RA [9]. Therefore, we suspect that variation in the immune response to a particular vaccine may depend on both the type of vaccine and which biological agent the patient had received. As intensive research in the field has led to the development of a number of new treatment options for patients with RA, it is essential to investigate the changes in immunogenicity following treatment in order to determine the optimal vaccination options to effectively ward off infection. For example, treatment modalities using calcineurin inhibitors have become available for treatment of RA diseases in the last decade [10]. This includes the $\mathrm{T}$ cell-specific immunosuppressant, tacrolimus (TAC). However, studies investigating the production of antibodies following pneumococcal vaccination in patients with RA diseases treated with TAC are limited [11, 12]. Thus, in this study, we have investigated the immunogenicity and tolerability of PPSV23 in Japanese patients with RA following treatment with TAC.

\section{Methods}

\section{Study design and patient population}

We performed a randomized, double-blind, controlled trial. Patients with clinically diagnosed RA were recruited in Japanese National Hospital Organization hospitals across Japan $(n=32)$ from September 2010 to
December 2012. Eligible patients were also found to be at risk for developing respiratory infections. Patients with RA were divided into the following groups: (1) patients with rheumatoid lung disease, (2) patients with RA treated with biological agents, and (3) patients who received immunosuppressive agents. Patients were excluded if they had previously received a pneumococcal vaccination. The following parameters were analyzed when the patient was first admitted to the study: swollen joint count, tender joint count, patient global assessment of disease activity, physician global assessment of disease activity, Health Assessment Questionnaire disability index (HAQ-DI) score, serum levels of C-reactive protein (CRP), and Disease Activity Score 28-joint assessment with CRP (DAS28 [CRP]) [13]. This study complies with the principles of the Declaration of Helsinki and was approved by the appropriate institutional review boards at each participating center. All patients provided written informed consent. This study was approved by the ethics committees of the National Hospital Organization central institutional review board (0512014, 2012) and was registered with University Hospital Medical Information Network Clinical Trials Registry (UMIN000009566).

\section{Intervention}

Patients were randomly assigned to receive either $0.5 \mathrm{ml}(25 \mu \mathrm{g})$ of PPSV23 (Pneumovax NP; Merck Sharp \& Dohme Corp., Tokyo, Japan) or $0.5 \mathrm{ml}$ of a placebo (sodium chloride) subcutaneously in the upper $\mathrm{arm}$. The vaccines were prepared in a masked fashion for those who administered it, blinding both the administrator of the vaccine and the patient to the type of vaccine given. Vaccine and placebo were presented in identical single-dose syringes and needle combinations that were labelled with sequential study numbers only. A statistician who was not on the study team carried out the randomization by using a random number table and numbered the containers accordingly.

Patients were instructed to record local reactions (e.g., redness, swelling, and tenderness) and systemic reactions (e.g., fever, nausea, and vomiting). Patients were also monitored for 12 months after enrollment to follow the development of pneumonia, including that stemming from pneumococcal disease. Serum samples were obtained immediately before and 4 to 6 weeks after vaccination and stored at $-30{ }^{\circ} \mathrm{C}$ until tested. An independent investigator measured the serotype-specific IgG geometric mean concentrations (GMCs) and opsonization indices (OIs) by using the sera samples from patients receiving PPSV23. The measurements were performed in random order, and all clinical data were blinded to prevent biases. 


\section{Enzyme-linked immunosorbent assays for serotype-specific IgG}

Enzyme-linked immunosorbent assays (ELISAs) for serotype-specific IgG were performed to measure the concentration of each type of antibody as previously described [9]. Furthermore, to measure IgG specificity for the $6 \mathrm{~B}$ and $23 \mathrm{~F}$ serotypes, we specifically performed our ELISAs according to the World Health Organization (WHO) standard procedure that used the international reference serum, 89SF-3 (graciously supplied by Carl E. Frasch). To improve the specificity of the assay, we performed a pneumococcal cell wall polysaccharide (C-PS) and pneumococcal $22 \mathrm{~F}$ polysaccharide pre-absorption step on the samples. The reference serum was preabsorbed with only C-PS [14, 15]. Detailed protocols are available at [16].

\section{Multiplexed opsonophagocytic assays}

To measure antibody functionality against pneumococcus, we performed multiplexed opsonophagocytic assays for pneumococcal serotypes $6 \mathrm{~B}$ and $23 \mathrm{~F}$ by using differentiated HL-60 cells and antibiotic-resistant target bacteria strains at the Research Institute for Microbial Disease, Osaka University, as previously described [17]. The quality control serum included in each assay was prepared from pooled sera of adults immunized with PPV23. The OI was defined as the serum dilution that led to $50 \%$ death of target bacteria. Opsotiter 3, an Excel-based data-processing program, was used to convert colony counts to OIs in accordance with the WHO protocol available at [18].

\section{Antibody response}

Fold increases relative to pre-vaccination values (ratios of post-vaccination value to pre-vaccination value) were determined. Positive antibody response was defined as at least a twofold increase in IgG concentrations or as at least a 10 -fold increase in OIs as described previously [9].

\section{Statistical analysis}

Clinical and demographic data are expressed as mean \pm standard deviation or as a percentage. Comparisons were made between the RA treatment groups before and after administration of the vaccine by using an analysis of variance with post-hoc Tukey's honesty significant difference test. Differences in IgG concentrations or OI before and after vaccination were compared by using the pairedsample $t$ test. Multivariate logistic regression analysis with adjustment for baseline characteristics was used to assess the relationship between positive antibody response to both pneumococcal serotype and a set of predictor variables, including age, gender, RA duration, current MTX, prednisolone use, TAC use, and serum IgG levels. A backward stepwise selection procedure was used to select significant independent variables. For all tests, probability values ( $P$ values) of less than 0.05 were considered statistically significant. All calculations were performed by using Excel Statistical Analysis 2008 (SSRI Co., Ltd., Tokyo, Japan) or PASW Statistics version 20 (SPSS Japan Inc., Tokyo, Japan).

\section{Results}

\section{Clinical and demographic characteristics}

In total, 989 subjects were assessed for eligibility, and 929 patients were recruited and randomly assigned (Additional file 1: Figure S1). Paired serum samples were obtained before and after vaccination from 703 subjects, 353 of which received PPV23. Patients receiving PPSV23 were divided into the following three groups according to their ongoing anti-RA therapy: TAC monotherapy $(2.0 \pm 0.7 \mathrm{mg} /$ day, TAC group, $n=29)$, MTX monotherapy $(7.8 \pm 2.4 \mathrm{mg} /$ week, MTX group, $n=55$ ), and DMARD (salazosulfapyridine $14 / 35$, bucillamine $5 / 35$, penicillamine $1 / 35$, sodium aurothiomalate $1 / 35$, ignatimod $1 / 35)$ or steroid $(13 / 35)$ treatments (RA control group; $n=35$ ). Patients' clinical and demographic characteristics are shown in Table 1. All participants fulfilled the criteria of safety required for vaccine injection, and no serious side effects were observed after vaccination.

\section{Serotype-specific IgG concentrations}

Four to six weeks after pneumococcal vaccination, the GMCs of both serotype 6B- and 23F-specific IgG were increased in all three groups $(P<0.00001$, Table 2$)$. However, these elevated GMC responses were not observed in the placebo group (Additional file 2: Table S1). Furthermore, the post-vaccination GMC of serotype 6B-specific antibodies was not significantly different when the three treatment groups were compared, implying that a similar increase was observed regardless of TAC or MTX treatment. However, the concentration of serotype 23Fspecific antibodies post-vaccination was significantly higher in patients who received TAC compared with the MTX treatment group $(P=0.005)$.

\section{Opsonophagocytic killing assays}

The post-vaccination OIs increased significantly in all treatment groups. The ratios between pre- and post-vaccination are provided in Table 2 . Whereas there was no significant difference observed between the TAC and control groups, there were significant differences in the post-vaccination OIs specific to $6 \mathrm{~B}$ and $23 \mathrm{~F}$ between the MTX and TAC treatment groups, and the TAC treatment group had higher OIs than the MTX group.

\section{Antibody response rates}

The antibody response rates, given as the percentage of patients with a positive antibody response, for patients in the 
Table 1 Clinical and demographic characteristics of rheumatoid arthritis patients prior to pneumococcal vaccination

\begin{tabular}{|c|c|c|c|c|}
\hline & $\begin{array}{l}\text { RA control } \\
n=35\end{array}$ & $\begin{array}{l}\text { MTX group } \\
n=55\end{array}$ & $\begin{array}{l}\text { TAC group } \\
n=29\end{array}$ & $\begin{array}{l}\text { TAC + MTX group } \\
n=14\end{array}$ \\
\hline Male/female & $12 / 23$ & $11 / 44$ & $9 / 20$ & $1 / 13$ \\
\hline Age in years, mean $\pm S D$ & $70.54 \pm 10.84$ & $63.80 \pm 11.50$ & $69.28 \pm 9.87$ & $61.00 \pm 11.68$ \\
\hline Weight in $\mathrm{kg}$, mean $\pm \mathrm{SD}$ & $53.32 \pm 9.54$ & $52.87 \pm 11.83$ & $52.22 \pm 12.24$ & $50.84 \pm 9.42$ \\
\hline $\mathrm{BMI}$, mean $\pm \mathrm{SD}$ & $21.79 \pm 3.47$ & $21.69 \pm 3.69$ & $21.84 \pm 4.43$ & $21.51 \pm 3.23$ \\
\hline $\mathrm{RA}$ duration in years, mean $\pm \mathrm{SD}$ & $11.66 \pm 12.52$ & $14.11 \pm 10.90$ & $10.52 \pm 9.74$ & $12.14 \pm 7.07$ \\
\hline MTX dose in mg/week, mean \pm SD & - & $7.80 \pm 2.37$ & - & $8.29 \pm 3.22$ \\
\hline $\mathrm{TAC}$ dose in $\mathrm{mg} /$ day, mean $\pm \mathrm{SD}$ & - & - & $2.05 \pm 0.74$ & $1.64 \pm 0.57$ \\
\hline Use of prednisolone, number (\%) of patients & $21(60.0)$ & $30(54.5)$ & $21(72.4)$ & $6(42.9)$ \\
\hline Prednisolone dose in mg/day, mean \pm SD & $6.06 \pm 4.23$ & $4.98 \pm 2.97$ & $3.89 \pm 1.99$ & $3.00 \pm 1.26$ \\
\hline DAS28 (CRP), mean \pm SD & $2.79 \pm 1.17$ & $2.61 \pm 0.98$ & $2.79 \pm 1.23$ & $2.10 \pm 0.71$ \\
\hline $\mathrm{HAQ}-\mathrm{DI}$, mean $\pm \mathrm{SD}$ & $0.85 \pm 0.84$ & $0.62 \pm 0.76$ & $0.76 \pm 0.88$ & $0.53 \pm 0.41$ \\
\hline SDAl, mean \pm SD & $9.03 \pm 6.32$ & $8.15 \pm 7.33$ & $10.36 \pm 10.63$ & $4.60 \pm 4.06$ \\
\hline CDAl, mean $\pm S D$ & $7.83 \pm 5.40$ & $7.45 \pm 6.73$ & $9.68 \pm 10.51$ & $3.95 \pm 3.84$ \\
\hline IP (\%) & $6(17.1)$ & $7(12.7)$ & $13(44.8)$ & 0 \\
\hline COPD (\%) & $3(8.6)$ & $1(1.8)$ & $2(6.9)$ & 0 \\
\hline Smoking history (\%) & $3(8.6)$ & $5(9.1)$ & $3(10.3)$ & $1(7.1)$ \\
\hline
\end{tabular}

Data were obtained immediately before pneumococcal vaccination

$R A$ rheumatoid arthritis, MTX methotrexate, TAC tacrolimus, SD standard deviation, BMI body mass index, DAS28 disease activity score $28, C R P$ C-reactive protein, $H A Q-D /$ Health Assessment Questionnaire disability index score, SDAl simplified disease activity index, CDAl clinical disease activity index, IP interstitial pneumonia, COPD chronic obstructive pulmonary disease

Table 2 Concentrations of pneumococcal polysaccharide antigen serotype-specific lgG antibodies and opsonization indices in the rheumatoid arthritis treatment groups before and after 23-valent pneumococcal polysaccharide vaccination

\begin{tabular}{|c|c|c|c|c|c|}
\hline & & & MTX group $n=55$ & TAC group $n=29$ & $\begin{array}{l}P \text { values between } \\
\text { treatment groups }\end{array}$ \\
\hline \multicolumn{6}{|c|}{$\mathrm{lgG}$ GMCs, $\mu \mathrm{g} / \mathrm{ml}$} \\
\hline \multirow[t]{3}{*}{$6 \mathrm{~B}$} & Before & 0.84 (0.58 to 1.11$)$ & 1.42 (0.86 to 1.97$)$ & $1.61(1.11$ to 2.11$)$ & NS \\
\hline & After & $4.05(2.13 \text { to } 5.97)^{*}$ & $4.36(2.17 \text { to } 6.55)^{*}$ & $8.35(2.79 \text { to } 13.91)^{*}$ & NS \\
\hline & Fold increase & 2.38 (1.41 to 5.62$)$ & 1.75 (1.15 to 3.11$)$ & 2.04 (1.71 to 5.76$)$ & NS \\
\hline \multirow[t]{3}{*}{$23 \mathrm{~F}$} & Before & 1.17 (0.85 to 1.48$)$ & 1.79 (1.33 to 2.25$)$ & 1.38 (0.68 to 2.08$)$ & NS \\
\hline & After & $11.61(4.16 \text { to } 19.07)^{*}$ & $7.41(4.48 \text { to } 10.33)^{*}$ & $16.73(7.81 \text { to } 25.65)^{*}$ & NS \\
\hline & Fold increase & 3.36 (1.85 to 9.42$)$ & 2.00 (1.27 to 5.48$)$ & 7.63 (3.70 to 18.85$)$ & 0.005 (MTX vs. TAC) \\
\hline \multicolumn{6}{|c|}{ GM-Ols } \\
\hline \multirow[t]{3}{*}{$6 B$} & Before & 17.24 (10.96 to 23.53$)$ & 150.79 (14.85 to 286.74$)$ & 262.89 (56.54 to 469.25$)$ & NS \\
\hline & After & $981.15(407.24 \text { to } 1555.05)^{*}$ & $584.29(270.29 \text { to } 898.28)^{*}$ & $2026.54(1047.56 \text { to } 3005.51)^{*}$ & 0.002 (MTX vs. TAC) \\
\hline & Fold increase & 10.22 (1.92 to 79.48$)$ & 2.57 (1.22 to 22.40$)$ & 20.80 (3.86 to 70.38$)$ & NS \\
\hline \multirow[t]{3}{*}{$23 \mathrm{~F}$} & Before & $63.21(-6.79$ to 133.20$)$ & 52.11 (14.04 to 90.18$)$ & $164.57(-129.84$ to 458.98$)$ & NS \\
\hline & After & $713.49(307.97 \text { to } 1119.01)^{*}$ & $724.56(336.93 \text { to } 1112.19)^{*}$ & 1583.04 (773.21 to 2392.86$)^{*}$ & 0.013 (MTX vs. TAC) \\
\hline & Fold increase & 6.86 (2.50 to 27.14$)$ & 3.75 (1.47 to 38.32$)$ & 64.38 (11.59 to 231.22$)$ & 0.048 (MTX vs. TAC) \\
\hline
\end{tabular}

IgG GMCs and GM-Ols are expressed as the mean (95\% confidence interval). Fold increases are expressed as the median (interquartile range). Differences between pre- and post-vaccination GMCs of serotype-specific lgG were assessed by using a paired-sample $t$ test. The three treatment groups were compared by using Kruskal-Wallis test with a Scheffé post-hoc test

MTX methotrexate, TAC tacrolimus, GMC geometric mean concentration, GM-Ol geometric mean opsonization index, NS not significant

${ }^{*} P<0.00001$ compared with pre-vaccination IgG GMCs or GM-Ols 
TAC treatment group were comparable to those of the untreated RA control group for both serotype $6 \mathrm{~B}$ and $23 \mathrm{~F}$ (Fig. 1a). However, the serotype 6B- and 23F-specific antibody response rates were significantly higher in the TAC group (6B $86.2 \%$, 23F $93.1 \%$ ) compared with the MTX group (6B 56.4 \%, 23F $65.5 \%$; $P=0.006$ and $P=0.005$, respectively). Furthermore, the percentage of patients with a positive antibody response for both strains was significantly greater in the TAC treatment group $(79.3 \%)$ compared with the MTX group ( $50.9 \%, P=0.011$ ). For OIs specific to serotype $6 \mathrm{~B}$ and $23 \mathrm{~F}$ (Fig. 1b), the TAC group (6B $57.7 \%$, 23F $82.1 \%$ ) showed a higher antibody response rate than did the MTX group (6B 34.6 \%, $P=0.052$; 23F $44.2 \%$, $P=0.001)$. For both strains, a higher proportion of the TAC-treated patients responded to pneumococcal vaccination compared with the patients who received MTX; however, this difference was not significant (46.2\% compared with $25.0 \%$, respectively; $P=0.059$ ).

\section{Comparison between TAC monotreatment and TAC/MTX combination treatment}

Notably, TAC is used primarily as a monotherapy, and the combined use of TAC and MTX is limited in Japan, as highlighted in a recent patient survey from this country [19]. Therefore, we were not surprised that only 14 RA patients receiving both TAC and MTX were enrolled in this study (Table 1). When these patients are compared with those receiving TAC monotherapy, it appears that both groups had a significant increase in the GMC and OI of the $6 \mathrm{~B}$ and $23 \mathrm{~F}$ serotypes after PPSV23 vaccination (Table 3). However, the post-vaccination GMC and the OIs for both serotypes were significantly lower in the patients receiving TAC/MTX combination therapy compared with those receiving TAC monotherapy. Similarly, a lower proportion of RA patients receiving TAC/MTX combination therapy had a geometric mean titer increase greater than twofold for both serotypes or an increase in
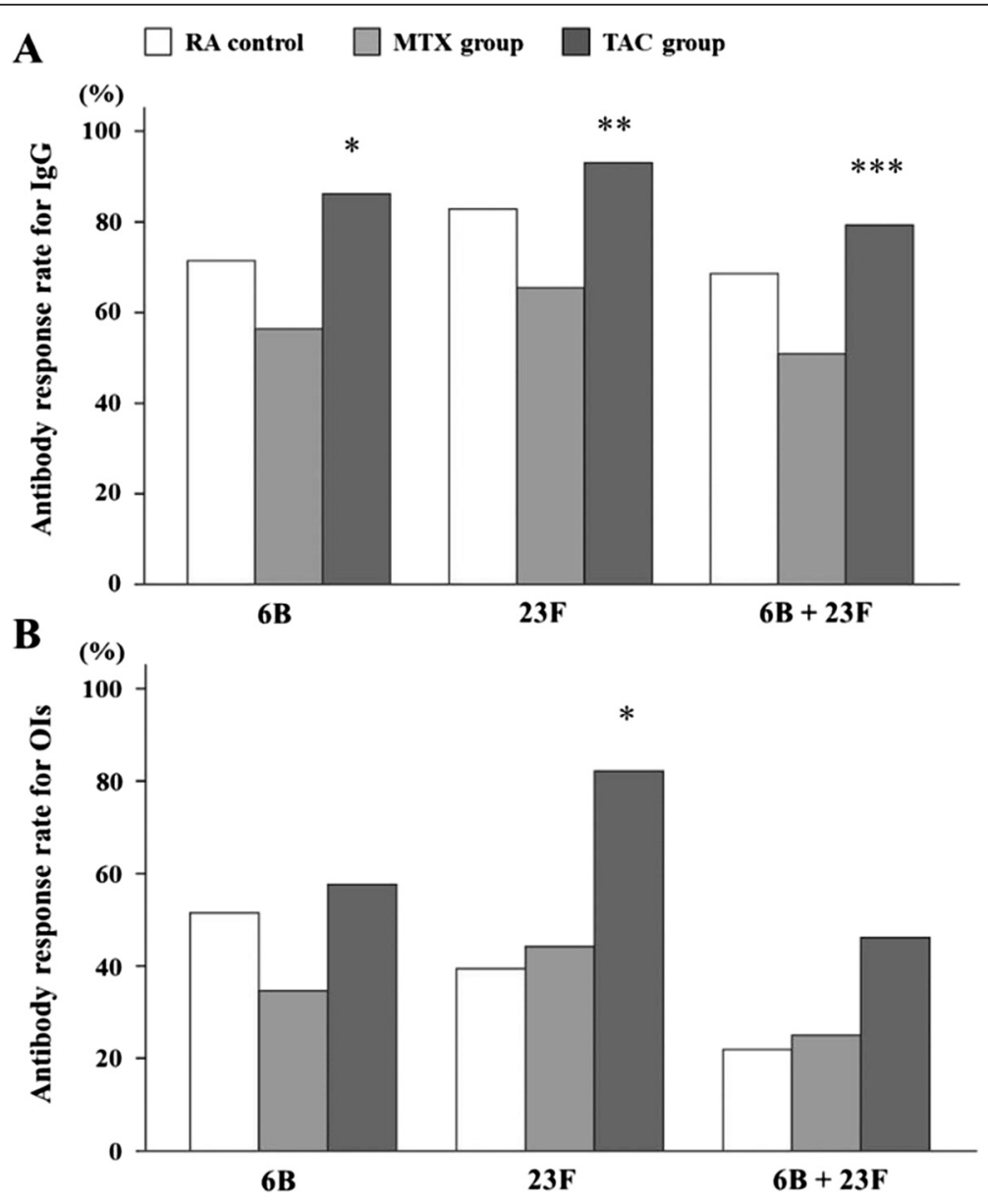

Fig. 1 Flow diagram of patient recruitment. Comparison of post-vaccination parameters in patients receiving TAC or MTX monotreatment. a Percentage of patients with an increase in $6 \mathrm{~B}$ and $23 \mathrm{~F}$ serotype-specific $\lg \mathrm{G}$ concentration greater than twofold. ${ }^{*} P=0.006,{ }^{* *} P=0.005,{ }^{* * *} P=0.011$ for TAC compared with MTX. b Percentage of patients with an increase in Ol for serotypes $6 \mathrm{~B}$ and $23 \mathrm{~F}$ greater than 10-fold. ${ }^{*} P=0.001$ (TAC versus RA control) and $P=0.001$ ( $T A C$ versus MTX). Data were compared by using the chi-squared test or Fisher's exact probability test. MTX methotrexate, Ol opsonization index, RA rheumatoid arthritis, TAC tacrolimus 
Table 3 Concentrations of pneumococcal polysaccharide antigen serotype-specific lgG antibodies and opsonization indices in the rheumatoid arthritis treatment groups before and after 23-valent pneumococcal polysaccharide vaccination

\begin{tabular}{|c|c|c|c|c|}
\hline & & TAC + MTX group $n=14$ & TAC group $n=29$ & $P$ value \\
\hline \multicolumn{5}{|c|}{$\operatorname{lgG} \mathrm{GMCs}, \mu \mathrm{g} / \mathrm{ml}$} \\
\hline \multirow[t]{3}{*}{$6 \mathrm{~B}$} & Before & 2.48 (0.92 to 4.04$)$ & $1.61(1.11$ to 2.11$)$ & 0.288 \\
\hline & After & $11.89(-2.25 \text { to } 26.03)^{*}$ & $8.35(2.79 \text { to } 13.91)^{* * *}$ & 0.551 \\
\hline & Fold increase & 1.39 (1.10 to 2.67$)$ & 2.04 (1.70 to 5.40$)$ & 0.023 \\
\hline \multirow[t]{3}{*}{$23 \mathrm{~F}$} & Before & 2.02 (0.93 to 3.11$)$ & 1.38 (0.68 to 2.08$)$ & 0.114 \\
\hline & After & $4.94(2.10 \text { to } 7.77)^{*}$ & $16.73(7.81 \text { to } 25.65)^{* * *}$ & 0.017 \\
\hline & Fold increase & 1.85 (1.14 to 3.82$)$ & 7.63 (3.70 to 18.85$)$ & $P<0.0001$ \\
\hline \multicolumn{5}{|c|}{ GM-Ols } \\
\hline \multirow[t]{3}{*}{$6 \mathrm{~B}$} & Before & 167.43 (34.41 to 300.45$)$ & 262.89 (56.54 to 469.25$)$ & 0.471 \\
\hline & After & $715.85(-138.24 \text { to } 1569.94)^{* *}$ & $2026.54(1047.56 \text { to } 3005.51)^{* * *}$ & 0.043 \\
\hline & Fold increase & 5.50 (2.31 to 10.60$)$ & 20.80 (3.86 to 70.38$)$ & 0.053 \\
\hline \multirow[t]{3}{*}{$23 \mathrm{~F}$} & Before & 9.50 (2.60 to 16.40$)$ & $164.57(-129.84$ to 458.98$)$ & 0.227 \\
\hline & After & $91.14(-13.42 \text { to } 195.70)^{*}$ & $1583.04(773.21 \text { to } 2392.86)^{* * *}$ & $P<0.0001$ \\
\hline & Fold increase & $3.51(1.00$ to 8.00$)$ & 64.38 (11.59 to 231.22$)$ & $P<0.0001$ \\
\hline
\end{tabular}

IgG GMCs and GM-Ols are expressed as the mean (95\% confidence interval). Fold increases are expressed as the median (interquartile range). Differences between pre- and post-vaccination GMCs of serotype-specific lgG were assessed by using a paired-sample $t$ test. $P$ values were calculated with chi-squared test for qualitative data TAC tacrolimus, MTX methotrexate, GMC geometric mean concentration, GM-Ol geometric mean opsonization index

${ }^{*} P<0.005,{ }^{* *} P<0.001,{ }^{* * *} P<0.00001$, compared with pre-vaccination IgG GMCs or GM-Ols

OI greater than 10-fold for the 23F serotype (Fig. 2). These data indicate that the combination of TAC and MTX treatment may cause reduced immune responsiveness against the pneumococcal vaccine PPSV23.

\section{Predictive factors for antibody response to PPSV23}

In a multivariate logistic regression analysis, TAC use was not identified as the predictive factor for antibody response to pneumococcal vaccination for either IgG concentrations or OIs (Additional file 3: Table S2, Additional file 4: Table S3). The negative association of current MTX use with antibody response was confirmed for both IgG concentrations specific to serotypes $6 \mathrm{~B}$ and $23 \mathrm{~F}$ (odds ratio $0.297,95 \%$ confidence interval 0.129 to 0.684 , $P=0.004$, Additional file 3: Table S2).

\section{Discussion}

Pneumococcal vaccination has been advocated for immunocompromised patients receiving the immunosuppressive RA treatments, such as TAC [20]. TAC is a type of calcineurin inhibitor that blocks T-cell cytokine production and indirectly affects B-cell activation [21]. Thus, a slight decrease in antibody production could be expected from this type of treatment. Notably, TAC has been used previously to prevent organ rejection following transplant and graft-versus-host disease after bone marrow transplants as well as for the treatment of myasthenia gravis, lupus nephritis, and ulcerative colitis [22]. However, the effects of TAC on the immunogenicity of pneumococcal vaccination are inconsistent in transplant recipients receiving treatment [23-25]. For example, Broeders et al. [26] previously assessed the immunogenicity of a polysaccharide vaccine in renal transplant recipients receiving TAC treatment and demonstrated that, although the protective effects of the vaccine were variable, all patients collectively produced suboptimal responses [26]. We suspect that these inconsistencies may be accounted for by the intrinsic effect of B-cell function under these treatment conditions.

In April 2005, TAC was approved as a treatment option for Japanese RA patients who had responded inappropriately to conventional treatments; this approval was subsequently granted in Canada, Korea, and Hong Kong [27]. However, our understanding concerning the interaction and effects of this treatment on vaccine function is limited. Therefore, to evaluate the immune response to PPSV23 in RA patients receiving TAC, we monitored several immune response parameters, including patient health, antibody concentration, and OI, following PPSV23 administration to patients receiving TAC and MTX. To this end, we examined the GMC of serotype $6 \mathrm{~B}$ and $23 \mathrm{~F}$ antibodies pre-vaccination and 4 to 6 weeks post-vaccination and found that both serotypes increased significantly in untreated and treated (TAC and MTX monotherapy) patients. It also appears that, for certain serotypes, this PPSV23 vaccine evoked a greater immunogenic response (i.e., a higher concentration and functional response) in RA patients receiving TAC compared with those receiving MTX. These 

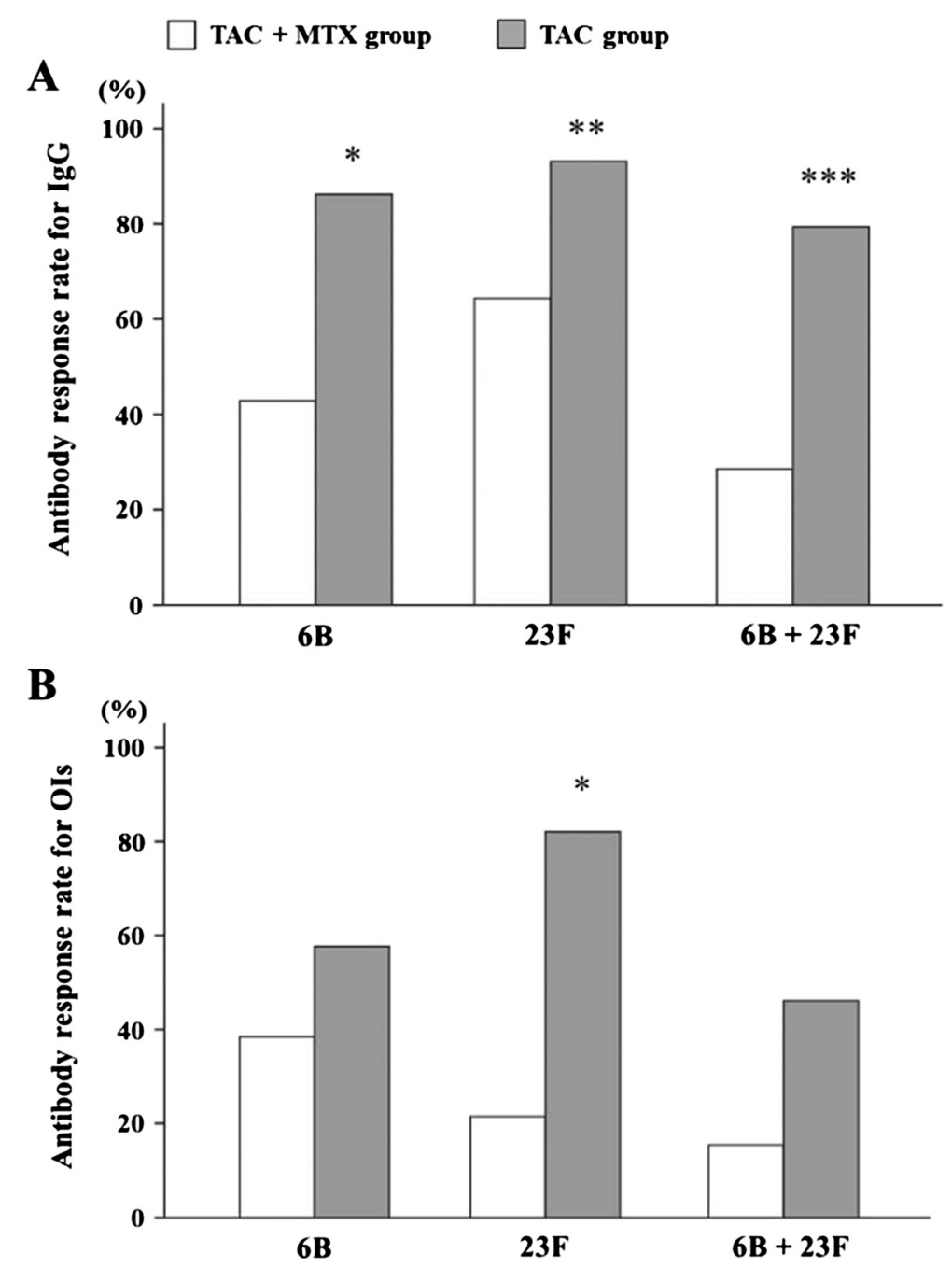

Fig. 2 Comparison of post-vaccination parameters in patients receiving TAC monotreatment or TAC/MTX combination treatment. a Percentage of patients with an increase in $6 \mathrm{~B}$ and $23 \mathrm{~F}$ serotype-specific $\lg \mathrm{G}$ concentration greater than twofold. ${ }^{*} P=0.005,{ }^{* *} P=0.028$, ${ }^{* *} P=0.001$, for TAC compared with TAC/MTX. b Percentage of patients with an increase in Ol for serotypes $6 \mathrm{~B}$ and $23 \mathrm{~F}$ greater than 10 -fold. ${ }^{*} P<0.0001$ for TAC compared with TAC/ MTX. Data were compared by using the chi-squared test or Fisher's exact probability test. MTX methotrexate, Ol opsonization index, TAC tacrolimus

findings suggest that the immunological response against $\mathrm{T}$ cell-independent polysaccharide antigens in RA patients following TAC-based immunosuppressive therapy is preserved in comparison with conventional MTX therapy. Combination therapy using both TAC and MTX had a decreased immune response to the PPSV23 vaccine in comparison with TAC alone. Taken together, these data indicate that a greater proportion of TAC-treated patients responded to PPSV23 more efficiently compared with patients who received MTX alone or in conjunction with TAC.

Notably, the pneumococcal vaccine used in this study consists almost exclusively of capsular polysaccharides [28]. This is important to note, as antibody production by B cells often requires "help" from T cells; however, highly repetitive polysaccharide structures have been shown to induce $\mathrm{T}$ cell-independent antibody production in B cells [29]. Thus, we suspect that the therapeutic immunosuppression observed in the TAC-treated RA patients in this study affects mainly T-cell function as this would not significantly alter the $\mathrm{T}$ cell-independent immune response to this specific pneumococcal vaccination. Furthermore, this conclusion is corroborated by other studies in this field [30]; however, additional work should be conducted by using vaccines with less repetitive polysaccharide structures. Immunogenicity of tetanus toxoid and 23-valent pneumococcal vaccinations was impaired in healthy subjects receiving abatacept, a selective T-cell co-stimulation modulator [31]. Also, treatment with abatacept was associated with diminished 
antibody response of pneumococcal conjugate vaccine (PCV 7) [8]. Our data were not consistent with this previous study by Tay et al., who used the same PPSV23 vaccination [31]. The differences in the subjects (healthy subjects versus RA patients) or $\mathrm{T}$ cell-targeted treatments (abatacept versus tacrolimus) may contribute to the discrepancies between our data and those of a previous study [31]. Protein-conjugated vaccines, which induce $\mathrm{T}$ cell-dependent immune response, may induce superior immune responses compared with the polysaccharide vaccine. However, further investigations for the immunogenicity of these $\mathrm{T}$ cell-dependent vaccines under $\mathrm{T}$ cell-targeted immunosuppressive treatments will be needed.

The primary limitation of this study is the relatively small number of patients with RA in each group, particularly for TAC/MTX combination treatment $(n=14)$. Sample size calculations were based on our previous study obtained RA patients receiving PPSV23 vaccination [9]. In this study, RA patients receiving MTX achieved the fold induction of $6 \mathrm{~B}$-specific (mean $\pm \mathrm{SD}$; $1.6 \pm 0.8$ ) and 23F-specific (mean \pm SD; $2.1 \pm 1.0$ ) IgG after 4 to 6 weeks from vaccination. Under this assumption, 33 (for 6B) or 27 (for 23F) evaluable patients samples for each group were needed with a one-tailed $t$ test with significance of 0.05 and $80 \%$ power to detect $40 \%$ reduction of GMC fold induction between the MTX group and the TAC/MTX group. Furthermore, we choose to investigate serotypes $6 \mathrm{~B}$ and $23 \mathrm{~F}$ because they are the main causative serotypes of penicillin-resistant pneumococcal pneumonia in Japan [32]. Although this allowed us to focus solely on these important serotypes, the effects of TAC on other serotypes during the PPSV23 vaccine-induce immune response are still unknown. Lastly, the antibody concentrations necessary for protection against invasive pneumococcal disease in adults have not been clearly defined [33]. Although we used the thresholds provided by previous studies, namely a twofold increase in IgG concentration and a 10-fold increase in the OI, to measure the positive antibody response to PPSV23 in this study, the suitability of these thresholds to predict prevention of pneumococcal infection has not been widely investigated.

\section{Conclusions}

We have shown that pneumococcal vaccination for RA patients receiving TAC treatment induced an adequate immunogenic response to the PPSV23 vaccine. However, diminished responsiveness to PPSV23 was observed in patients receiving TAC and concomitant MTX. On the basis of these data, we believe that pneumococcal vaccination and TAC treatment should be used more frequently in infection-prone patients with RA.

\section{Additional files}

Additional file 1: Figure S1. Flow diagram of patient recruitment.

Additional file 2: Table S1. Concentrations of pneumococcal polysaccharide antigen serotype-specific lgG antibodies and opsonization indices in the rheumatoid arthritis (RA) patients before and after placebo injection.

Additional file 3: Table S2. Predictors of positive lgG response for both $6 \mathrm{~B}$ and $23 \mathrm{~F}$ among patients with rheumatoid arthritis (RA).

Additional file 4: Table S3. Predictors of positive opsonization index $(O /)$ response for both $6 \mathrm{~B}$ and $23 \mathrm{~F}$ among patients with rheumatoid arthritis $(R A)$.

\section{Abbreviations}

C-PS: Cell wall polysaccharide; CRP: C-reactive protein; DMARD: Disease-modifying antirheumatic drug; ELISA: Enzyme-linked immunosorbent assay; GMC: Geometric mean concentration; MTX: Methotrexate; Ol: Opsonization index; PPSV23: 23-valent pneumococcal polysaccharide vaccine; RA: Rheumatoid arthritis; TAC: Tacrolimus; TNF: Tumor necrosis factor; WHO: World Health Organization.

\section{Competing interests}

The authors declare that they have no competing interests.

\section{Authors' contributions}

KM, KK, TMa, HF, and KO participated in the design of the study. ST participated in the design of the study and helped to collect the clinical data. MA participated in the design of the study and helped to analyze the data. YA helped to analyze the data. FH, HI, RM, ES, TMi, SM, TF, YI, NI, HT, KS, TY, SO, TS, YK, MK, YS, AO, HO, YO, Kl, and SY helped to collect the clinical data. All authors wrote the manuscript and read and approved the final manuscript.

\section{Acknowledgments}

The study was supported by research grants from the Ministry of Health, Labour and Welfare of Japan and research funds from the National Hospital Organization (NHO), Japan. The authors are grateful to Michiyo Hayakawa and Yumi Hattori (RIMD, Osaka University), Yuka Koizumi (RIMD, Osaka University), and Michiyo Hayakawa (RIMD, Osaka University) for technical assistance in measuring serotype-specific lgG concentrations and Ols. This study could not have been completed without the effective and dedicated participation of each of the following contributors: Naoya Mori (NHO Shinshu Ueda Medical Center), Akinori Matsumori (NHO Kochi Hospital), Koichiro Takahi (NHO Toneyama Hospital), Kiyoki Kitagawa (NHO Kanazawa National Hospital), Tetsuo Ozawa (NHO Niigata Hospital), Norikazu Hamada (NHO Kure Medical Center), Kyoichi Nakajima (NHO Higashisaitama Hospital), Norio Tamura (NHO Nishitaga Hospital), Takashi Oikawa (NHO Hatinohe Hospital), Hideaki Nagai (NHO Tokyo Hospital), Yasuhiro Komiya (NHO Shigaraki Hospital), and Masaharu Kawabata (NHO Minami Kyushu Hospital).

\section{Author details}

${ }^{1}$ Japanese National Hospital Organization (NHO, EBM study group), Higashigaoka 2-5-23, Meguro, Tokyo 152-8621, Japan. ${ }^{2}$ Clinical Research Center, NHO Nagasaki Medical Center, Kubara 2-1001-1, Omura 856-8652, Japan. ${ }^{3}$ Research Institute for Microbial Diseases, Osaka University, Yamadaoka 3-1, Suita, Osaka 565-8563, Japan. ${ }^{4}$ Department of Public Health and Epidemiology, Meiji Pharmaceutical University, Noshio 2-522-1, Kiyose, Tokyo 204-8588, Japan. Infectious Diseases Surveillance Center, National Institute of Infectious Diseases, Toyama 1-23-1, Shinjuku, Tokyo 162-8640, Japan.

Received: 19 December 2014 Accepted: 26 May 2015

Published online: 03 June 2015

\section{References}

1. Segal BH, Sneller MC. Infectious complications of immunosuppressive therapy in patients with rheumatic diseases. Rheum Dis Clin North Am. 1997;23:219-37. 
2. Glück T, Müller-Ladner U. Vaccination in patients with chronic rheumatic or autoimmune diseases. Clin Infect Dis. 2008:46:1459-65.

3. Brezinschek HP, Hofstaetter T, Leeb BF, Haindl P, Graninger WB. Immunization of patients with rheumatoid arthritis with antitumor necrosis factor alpha therapy and methotrexate. Curr Opin Rheumatol. 2008;20:295-9.

4. van Assen S, Agmon-Levin N, Elkayam O, Cervera R, Doran MF, Dougados $M$, et al. EULAR recommendations for vaccination in adult patients with autoimmune inflammatory rheumatic diseases. Ann Rheum Dis. 2011;70:414-22.

5. van Assen S, Elkayam O, Agmon-Levin N, Cervera R, Doran MF, Dougados $M$, et al. Vaccination in adult patients with auto-immune inflammatory rheumatic diseases: a systematic literature review for the European League Against Rheumatism evidence-based recommendations for vaccination in adult patients with auto-immune inflammatory rheumatic diseases. Autoimmun Rev. 2011;10:341-52.

6. Kapetanovic MC, Saxne T, Sjöholm A, Truedsson L, Jönsson G, Geborek P. Influence of methotrexate, TNF blockers and prednisolone on antibody responses to pneumococcal polysaccharide vaccine in patients with rheumatoid arthritis. Rheumatology (Oxford). 2006;45:106-11.

7. Hua C, Barnetche T, Combe B, Morel J. Effect of methotrexate, anti-tumor necrosis factor $a$, and rituximab on the immune response to influenza and pneumococcal vaccines in patients with rheumatoid arthritis: a systematic review and meta-analysis. Arthritis Care Res (Hoboken). 2014;66:1016-26.

8. Crnkic Kapetanovic M, Saxne T, Jönsson G, Truedsson L, Geborek P. Rituximab and abatacept but not tocilizumab impair antibody response to pneumococcal conjugate vaccine in patients with rheumatoid arthritis. Arthritis Res Ther. 2013;15:R171.

9. Mori S, Ueki Y, Akeda Y, Hirakata N, Oribe M, Shiohira Y, et al. Pneumococcal polysaccharide vaccination in rheumatoid arthritis patients receiving tocilizumab therapy. Ann Rheum Dis. 2013;72:1362-6.

10. Kaminuma O. Selective inhibitors of nuclear factor of activated T cells: potential therapeutic drugs for the treatment of immunological and inflammatory diseases. Inflamm Allergy Drug Targets. 2008;7:35-40.

11. Stiehm ER, Roberts RL, Kaplan MS, Corren J, Jaracz E, Rico MJ. Pneumococcal seroconversion after vaccination for children with atopic dermatitis treated with tacrolimus ointment. J Am Acad Dermatol. 2005;53:S206-13.

12. Lindemann M, Heinemann FM, Horn PA, Witzke O. Immunity to pneumococcal antigens in kidney transplant recipients. Transplantation. 2010;90:1463-7.

13. van Gestel AM, Prevoo ML, Van't Hof MA, van Rijswijk MH, van de Putte LB, van Riel PL. Development and validation of the European League against Rheumatism response criteria for rheumatoid arthritis. Comparison with the preliminary American College of Rheumatology and the World Health Organization/International League against Rheumatism Criteria. Arthritis Rheum. 1996:39:34-40.

14. Concepcion NF, Frasch CE. Pneumococcal type $22 f$ polysaccharide absorption improves the specificity of a pneumococcal-polysaccharide enzyme-linked immunosorbent assay. Clin Diagn Lab Immunol. 2001;8:266-72.

15. Wernette CM, Frasch CE, Madore D, Carlone G, Goldblatt D, Plikaytis B, et al. Enzyme-linked immunosorbent assay for quantitation of human antibodies to pneumococcal polysaccharides. Clin Diagn Lab Immunol. 2003;10:514-9.

16. World Health Organization Pneumococcal Serology Reference Laboratories at the Institute of Child Health, University College London, UK, and the Department of Pathology, University of Alabama, Birmingham, AL, USA. Training manual for Enzyme linked immunosorbent assay for the quantitation of Streptococcus pneumoniae serotype specific lgG (Pn PS ELISA). www.vaccine.uab.edu/ELISAProtocol(89SF).pdf.

17. Burton RL, Nahm MH. Development and validation of a four fold multiplexed opsonization assay (MOPA4) for pneumococcal antibodies. Clin Vaccine Immunol. 2006;13:1004-9.

18. Nahm MH, Burton RL. Protocol for multiplexed opsonophagocytic killing assay (UAB-MOPA) for antibodies against Streptococcus pneumoniae. www.vaccine.uab.edu/UAB-MOPA.pdf.

19. Takeuchi T, Kawai S, Yamamoto K, Harigai M, Ishida K, Miyasaka N. Post-marketing surveillance of the safety and effectiveness of tacrolimus in 3,267 Japanese patients with rheumatoid arthritis. Mod Rheumatol. 2014;24:8-16.

20. Simberkoff MS, Cross AP, Al-Ibrahim M, Baltch AL, Geiseler PJ, Nadler J, et al. Efficacy of pneumococcal vaccine in high-risk patients. Results of a Veterans Administration Cooperative Study. N Engl J Med. 1986;315:1318-27.

21. Sigal NH, Dumont FJ. Cyclosporin A, FK-506, and rapamycin: pharmacologic probes of lymphocyte signal transduction. Annu Rev Immunol. 1992;10:519-60.
22. Singer NG, McCune WJ. Update on immunosuppressive therapy. Curr Opin Rheumatol. 1998;10:169-73.

23. Kumar D, Rotstein C, Miyata G, Arlen D, Humar A. Randomized, double-blind, controlled trial of pneumococcal vaccination in renal transplant recipients. J Infect Dis. 2003;187:1639-45.

24. Lindemann $M$, Heinemann FM, Horn PA, Witzke O. Long-term response to vaccination against pneumococcal antigens in kidney transplant recipients. Transplantation. 2012;94:50-6.

25. Kumar D, Welsh B, Siegal D, Chen MH, Humar A. Immunogenicity of pneumococcal vaccine in renal transplant recipients-three year follow-up of a randomized trial. Am J Transplant. 2007;7:633-8.

26. Broeders EN, Wissing KM, Ghisdal L, Lemy A, Hoang AD, Vereerstraeten P, et al. Large decrease of anti-tetanus anatoxin and anti-pneumococcal antibodies at one year after renal transplantation. Clin Nephrol. 2013;79:313-7.

27. Kitahara K, Kawai S. Cyclosporine and tacrolimus for the treatment of rheumatoid arthritis. Curr Opin Rheumatol. 2007;19:238-45.

28. Jackson LA, Janoff EN. Pneumococcal vaccination of elderly adults: new paradigms for protection. Clin Infect Dis. 2008;47:1328-38.

29. Lee CJ, Lee LH, Frasch CE. Protective immunity of pneumococcal glycoconjugates. Crit Rev Microbiol. 2003;29:333-49.

30. Mond JJ, Lees A, Snapper CM. T cell-independent antigens type 2. Annu Rev Immunol. 1995;13:655-92.

31. Tay L, Leon F, Vratsanos G, Raymond R, Corbo M. Vaccination response to tetanus toxoid and 23-valent pneumococcal vaccines following administration of a single dose of abatacept: a randomized, open-label, parallel group study in healthy subjects. Arthritis Res Ther. 2007;9:R38.

32. Oishi $\mathrm{K}$, Yoshimine $\mathrm{H}$, Watanabe $\mathrm{H}$, Watanabe $\mathrm{K}$, Tanimura $\mathrm{S}$, Kawakami $\mathrm{K}$, et al. Drug-resistant genes and serotypes of pneumococcal strains of community-acquired pneumonia among adults in Japan. Respirology. 2006;11:429-36.

33. Dransfield MT, Nahm MH, Han MK, Harnden S, Criner GJ, Martinez FJ, et al. Superior immune response to protein-conjugate versus free pneumococcal polysaccharide vaccine in chronic obstructive pulmonary disease. Am J Respir Crit Care Med. 2009;180:499-505.

\section{Submit your next manuscript to BioMed Central and take full advantage of:}

- Convenient online submission

- Thorough peer review

- No space constraints or color figure charges

- Immediate publication on acceptance

- Inclusion in PubMed, CAS, Scopus and Google Scholar

- Research which is freely available for redistribution 